\title{
Location-Based Coverage Probability for Distributed Antenna Systems in Finite-Area Networks
}

\author{
Mohammud Z. Bocus ${ }^{1}$, Orestis Georgiou ${ }^{1}$, Justin P. Coon ${ }^{2}$ and Dene A. Hedges ${ }^{2}$ \\ 1 Toshiba Telecommunications Research Laboratory, 32 Queen Square, Bristol, BS1 4ND, UK. \\ 2 Department of Engineering Science, University of Oxford, Parks Road, OX1 3PJ, Oxford, UK.
}

\begin{abstract}
The performance of distributed wireless communication systems is dictated by the system boundaries and the interference regimes. In this paper, we present a novel coverage and connectivity analysis of a wireless system within confined domains. Specifically, a system where the receiver connects to the nearest transmitter is analyzed. Using tools from stochastic geometry, we derive general expressions quantifying the dependence of the system performance on the location of the receiver for general geometries, inclusive of circles and rectangles. The developed theory and expressions provide new insights on where additional nodes must be deployed in an existing network in order to maximize a desired quality of service. We further extend the analysis to networks with multiple collaborating transmitters which use maximum ratio transmission (MRT) or joint transmission such as in CoMP and other distributed antenna systems. Our results indicate that while having more collaborating transmitters can improve the performance of the system, artefacts due to the domain geometries cannot be eliminated. We corroborate our analysis through simulations.
\end{abstract}

\section{INTRODUCTION}

The fifth generation of wireless communication systems is envisaged to provide enhanced throughput and quality of service as well as significantly reducing latency and energy consumption. To this end, the potentials of several candidate technologies have been investigated [1]. One promising technology is extreme network densification. Densification can be enabled through distributed antenna systems, self-organizing networks and advanced interference management such as in cloud-RAN systems, among others (see [2] and references therein). From the point of view of network designers, having a structured method to analyze and optimize such systems is desirable.

The performance of dense networks has been an area of active research for the past few years [3], [4]. Tools from stochastic geometry have often been used to facilitate the analysis under different network environments. Conventionally, the modeling of the deployment of devices and/or users as Poisson point processes (PPP) has ensured some tractable mathematics. While initial studies have considered mainly infinite domains, it has been realized that the performance of networks, in practice, can be severely impaired by geometric boundary effects, e.g., indoors. In [5], for instance, the authors investigate the performance of access point deployment in finite areas and show that the signal-to-interference-and-noise-ratio (SINR) under the assumption of finite and infinite domains can differ considerably. Their study is however limited to circular domains.
In this paper, we present a generic framework for analyzing interference limited systems in finite domains. We derive the connection probability for a system where each receiver connects to the transmitter nearest to it. Such a scenario can for instance represent the deployment of several 802.11 access points (APs) in an area, with each AP serving the nearest user. Transmitters not associated to a particular receiver contribute to interference, assuming all APs operate on the same channel. In this paper, we will use the term AP and transmitter interchangeably. Our analysis illustrates how the performance of typical systems is affected by the location of the receiver within the domain. We explain how the coverage probability can be derived in any complex geometry and provide examples for rectangular and circular domains. The latter can for example model a large indoor stadium environment. We also present the coverage analysis for large domains where local effects have a larger impact on the system performance. Although the derived expressions are in semi-analytic form, they can be readily evaluated using common software such as Matlab or Mathematica. The analysis and results point to an annular region close to the domain boundaries where the coverage probability is higher. This observation suggests that without a priori knowledge of existing nodes positions, additional nodes should be deployed in the neighborhood of these annular regions for improved performance.

We further extend the analysis to scenarios where the receiver connects to multiple transmitters, such as in the cooperative multipoint (CoMP) scheme of LTE. We limit our analysis to the maximum ratio transmission (MRT) scheme. While some recent papers have analyzed the performance of transmitter cooperation in large domains, e.g., [4], [6][8], none, to the authors' knowledge, have considered border effects. In [8] for instance, a model to characterize the signalto-interference ratio of a typical downlink system under zeroforcing beamforming and non-coherent joint transmission is presented for unbounded systems. This paper aims to provide new insights on cooperative transmission schemes in finite geometries, exemplifying the importance of boundary effects and their mitigation, and encourage further analysis of other cooperative or multiple antenna communication schemes. To summarize, the main contributions of this paper are (1) a generic framework for analyzing the coverage of finite domain systems, (2) a novel expression for large domain analysis and (3) the coverage analysis in cooperative transmission systems employing MRT. 
The remainder of the paper is structured as follows. The system model is presented in Section II. The coverage and connectivity analysis are presented in Sections III and IV, respectively. The analysis of cooperating communications is given in V. Finally, some concluding remarks are provided in Section VI.

\section{Network Definitions And System Model}

Consider a $n \geq 2$ dimensional PPP with intensity $\rho$ inside some subset $\mathcal{V} \subset \mathbb{R}^{n}$ and of zero intensity in $\mathbb{R}^{n} \backslash \mathcal{V}$. Let these points represent single-antenna APs which are constantly transmitting with power $\mathcal{P}$. We let the coordinates of access point $i$ be denoted by $\mathbf{t}_{i} \in \mathcal{V}$ and relabel them relative to a receiver $\mathbf{r} \in \mathcal{V}$ in ascending order of separation such that $d_{1} \leq d_{2} \leq d_{3} \leq \ldots$ where $d_{i}=\left|\mathbf{t}_{i}-\mathbf{r}\right|$ is the Euclidean distance between AP $i$ and the receiver at $\mathbf{r}$.

In this work, we analyze the performance of a system where a receiver associates with the transmitter nearest to it. Using the same definitions as in [9], the SINR for a receiver at $\mathbf{r}$ is expressed as

$$
\operatorname{SINR}_{1}=\frac{\mathcal{P}\left|h_{1}\right|^{2} g\left(d_{1}\right)}{\sigma^{2}+\gamma \mathcal{I}_{1}},
$$

where $\sigma^{2}$ is the noise power, $h_{i}$ represents the channel between the $i$ th nearest AP and the receiver, $g(d)=\frac{1}{\epsilon+d^{\eta}}, \epsilon \geq 0$ accounts for the path loss at a separation of $d$ and path loss exponent $\eta$ and $\mathcal{I}_{1}$ is the total interference observed at $\mathbf{r}$ which can be approximated as [8]

$$
\mathcal{I}_{1} \approx \mathcal{P} \sum_{k \neq 1}\left|h_{k}\right|^{2} g\left(d_{k}\right)
$$

Note that in practice, the interfering signals are non-coherent and will not always interfere constructively, i.e., the sum interference power will not be equal to the sum of individual interfering signal powers. However, it was shown in [8] that the above approximation is valid for orthogonal frequency division multiplexing (OFDM) systems. We make the same assumption in this paper. Similar to the system model in [9], the orthogonality factor $\gamma \in[0,1]$ denotes the proportion by which the desired signal is influenced by interference. An interference-free system is represented by $\gamma=0$, i.e., all signals are orthogonal. On the opposite extreme, $\gamma=1$ corresponds to a systems where all signals interfere with one another.

\section{CONNECTIVITy AnAlysis}

The connection probability is often used in wireless networks to study both infinite [10] and, more recently, finite area cellular networks [5], [9]. This metric can be interpreted as the complement of the well-known outage probability.

Conditioned on the distance to the nearest AP, the connection probability for a receiver at $\mathbf{r}$ can be expressed as

$$
\begin{aligned}
H_{1}\left(\mathbf{r}, d_{1}\right) & =\mathbb{P}\left[\mathrm{SINR}_{1} \geq q \mid \mathbf{r}, d_{1}\right] \\
& =\mathbb{P}\left[\left|h_{1}\right|^{2} \geq \frac{q\left(\sigma^{2}+\gamma \mathcal{I}_{1}\right)}{\mathcal{P} g\left(d_{1}\right)} \mid \mathbf{r}, d_{1}\right]
\end{aligned}
$$

where $q$ represents the SINR threshold. As opposed to studies that consider infinite PPPs on $\mathbb{R}^{n}$, it should be observed that (3) is strongly dependent on the position of the receiving node $\mathbf{r} \in \mathcal{V}$. Further conditioning on the interference realization $\mathcal{I}_{1}$ and assuming Rayleigh fading with $h_{i} \sim \mathcal{C N}(0,1)$, the connection probability is known to equate to [9]

$$
H_{1}\left(\mathbf{r}, d_{1}\right)=e^{-\frac{q \sigma^{2}}{\mathcal{P} g\left(d_{1}\right)}} \mathcal{L}_{\mathcal{I}_{1}}\left(\frac{q \gamma}{\mathcal{P} g\left(d_{1}\right)}\right)
$$

where

$$
\mathcal{L}_{\mathcal{I}_{1}}\left(s_{1} / \mathcal{P}\right)=\exp \left(-\rho \int_{\hat{\mathcal{V}}_{1}} \frac{s_{1} g\left(d_{k}\right)}{1+s_{1} g\left(d_{k}\right)} \mathrm{dt}_{k}\right),
$$

is the Laplace transform of the random variable $\mathcal{I}_{1}$ evaluated at $s_{1}=\frac{q \gamma}{g\left(d_{1}\right)}$ and conditioned on the location of the receiver $\mathbf{r}$ and its nearest AP at $\mathbf{t}_{1}$. The above equalities follow from the channel gains being independent of each other. This is true as long as APs are separated by at least half of a wavelength.

Note that (5) depends on the location of the receiver and its nearest AP through $\hat{\mathcal{V}}_{1}=\mathcal{V} \backslash B_{d_{1}}(\mathbf{r})$, where $B_{d_{1}}(\mathbf{r})$ refers to a ball of radius $d_{1}$ centered at $\mathbf{r}$. While no simple closedform expressions can be derived for (5), it can be evaluated numerically for any domain $\mathcal{V}$. In particular, such evaluations can be performed by defining the distance from the boundary of $B_{d_{1}}(\mathbf{r})$ within the domain to the boundary of the domain.

In the next subsection, we derive the connection probabilities for the special cases of circular and rectangular domains.

\section{A. Connection Probability for a Circular Domain}

If $\mathcal{V}$ is a two-dimensional circular domain of radius $R$, (5) can be simplified and expressed as an angular integral of the ${ }_{2} F_{1}$ Gauss hyper-geometric functions. The latter is a function of the receiver's radial distance $r$ from the center of the domain. The natural log of the above defined Laplace transform is given by

$\ln \mathcal{L}_{\mathcal{I}_{1}}\left(s_{1}\right)=-2 \rho \int_{0}^{\hat{\theta}} \phi\left(r \cos \theta+\sqrt{R^{2}-r^{2} \sin ^{2} \theta}\right)-\phi\left(d_{1}\right) \mathrm{d} \theta$

where $\hat{\theta}=\min \left[\left|\arccos \frac{r^{2}+d_{1}^{2}-R^{2}}{2 r d_{1}}\right|, \pi\right]$ and

$$
\begin{aligned}
\phi(y) & =\int_{0}^{y} \frac{s_{1} g(x)}{1+s_{1} g(x)} x \mathrm{~d} x \\
& =\frac{s_{1} y^{2}}{2\left(s_{1}+\epsilon\right)}{ }_{2} F_{1}\left(1, \frac{2}{\eta}, 1+\frac{2}{\eta},-\frac{y^{\eta}}{s_{1}+\epsilon}\right),
\end{aligned}
$$

which can be expressed in terms of elementary functions for $\eta=1,2$, and 4 [9]. Note that the arguments of the $\phi(\cdot)$ in (6) corresponds to the distance from a point of interest to the boundary of the domain. Such expressions can be easily derived using common rules of trigonometry and the derivations have been omitted herein for the sake of conciseness.

\footnotetext{
${ }^{1}$ The natural logarithm is used here for a more concise representation.
} 


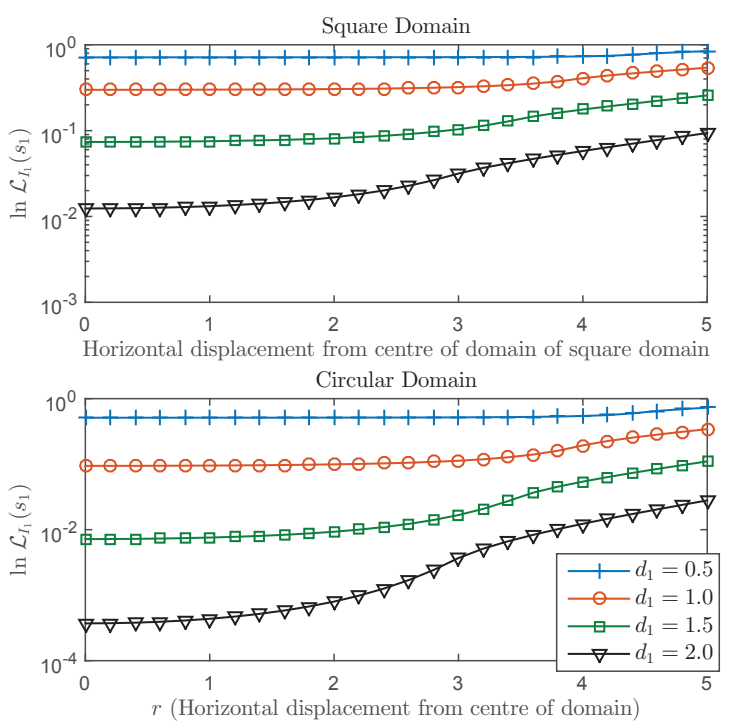

Fig. 1. Plots of $\mathcal{L}_{\mathcal{I}_{1}}\left(s_{1}\right)$ as a function of the displacement $r$ of the receiver from the origin of $\mathcal{V}$ using $\epsilon=0.01, \eta=4, R=5$ for the circle, $L=10$ for the square and $q=\mathcal{P}=\gamma=\sigma^{2}=1$; for the top plot, $y_{u}=0$.

\section{B. Connection Probability for a Rectangular Domain}

As opposed to a circular domain which offers rotational symmetry, defining the distance from the boundary of the ball $B_{d_{1}}(\mathbf{r})$ to the boundary of a rectangular/square domain requires some extra care. Consider a $2-\mathrm{D}$ rectangular domain of length $L$ and width $w$ for instance. Let the center of the domain be the origin and let the receiver be at position $\left(x_{u}, y_{u}\right)$. Using simple rules for calculating distances between 2 points, it is easy to verify that the Laplace transform is given by

$$
\begin{aligned}
\mathcal{L}_{I_{1}}(s)=-\rho \int_{0}^{2 \pi}[ & \left(\min \left(\left|\frac{\frac{L}{2}-\left|x_{u}\right|}{\cos \theta}\right|,\left|\frac{\frac{w}{2}-\left|y_{u}\right|}{\sin \theta}\right|\right)\right) \\
& \left.-\phi\left(d_{1}\right)\right]^{+} d \theta
\end{aligned}
$$

where $[x]^{+}=\max \{x, 0\}$.

Fig. 1 plots the Laplace transform $\mathcal{L}_{\mathcal{I}_{1}}\left(s_{1}\right)$ of the interference as a function of displacement $r$ of the receiver from the origin of $\mathcal{V}$ for different values of $d_{1}$ for a square and circle domain respectively. From the plot, it becomes evident that nodes are significantly more likely to be connected near the boundary, especially when the nearest AP is not so close by. As a consequence, interfering nodes are also further away.

\section{Coverage Analysis}

The coverage probability can be interpreted as the probability that a receiver located at $\mathbf{r}$ is in coverage, i.e., the probability that the received signal at $\mathbf{r}$ from its nearest AP satisfies $\mathrm{SINR}_{1} \geq q$. This metric is a functional of $H_{1}$ obtained by averaging over the distance $d_{1}$ to the nearest AP

$$
C_{1}(\mathbf{r})=\int_{0}^{\infty} H_{1}\left(\mathbf{r}, d_{1}\right) f_{1}\left(\mathbf{r}, d_{1}\right) \mathrm{d} d_{1}
$$

where $f_{1}\left(\mathbf{r}, d_{1}\right)$ is the probability density function (pdf) of the distance to the nearest AP given $\mathbf{r} \in \mathcal{V}$. It is worth mentioning here that the domain boundaries are implicitly considered in $f_{1}\left(\mathbf{r}, d_{1}\right)$. Thus, even though we are analyzing bounded domains, the upper limit of the integral in (9) can be set to infinity without affecting the results.

We can derive $f\left(\mathbf{r}, d_{1}\right)$ for a general domain $\mathcal{V}$ through the void probability in a ball $B_{d_{1}}(\mathbf{r})$ as

$$
\begin{aligned}
\mathbb{P}\left[d_{i} \geq d_{1}, \forall i \geq 1\right] & =\mathbb{P}\left[\text { No AP closer than } d_{1}\right] \\
& =\exp \left(-\rho\left|B_{d_{1}}(\mathbf{r}) \cap \mathcal{V}\right|\right) .
\end{aligned}
$$

The desired pdf is obtained by differentiating $-\mathbb{P}\left[d_{i} \geq d_{1}, \forall i \geq 1\right]$ once with respect to $d_{1}$. Note that the support of $f\left(\mathbf{r}, d_{1}\right)$ is from 0 to the longest distance from $\mathbf{r}$ to the domain border $d_{\max }(\mathbf{r})$.

In an unbounded domain $\left|B_{d_{1}}(\mathbf{r}) \cap \mathcal{V}\right|=\pi^{\frac{n}{2}} d_{1}^{n} / \Gamma\left(1+\frac{n}{2}\right)$ in $n \geq 1$ dimensions such that it is independent of $\mathbf{r}$

$$
f\left(d_{1}\right)=\frac{\rho \pi^{n / 2} n d_{1}^{n-1}}{\Gamma\left(1+\frac{n}{2}\right)} \exp \left(-\frac{\rho \pi^{\frac{n}{2}} d_{1}^{n}}{\Gamma\left(1+\frac{n}{2}\right)}\right) .
$$

In bounded domains, however, $\left|B_{d_{1}}(\mathbf{r}) \cap \mathcal{V}\right|$ is a piecewise function of $d_{1}$. It should be noted that bounded domains need not be finite, e.g., a positive half-plane in two dimensions. Hence, depending on the location of the receiver $\mathbf{r}$ relative to the domain border, for small values of $d_{1},\left|B_{d_{1}}(\mathbf{r}) \cap \mathcal{V}\right|=$ $\pi^{\frac{n}{2}} d_{1}^{n} / \Gamma\left(1+\frac{n}{2}\right)$ as in the unbounded case above. This is the regime corresponding to the ball $B_{d_{1}}(\mathbf{r})$ not intersecting the domain border. As $d_{1}$ increases, the ball $B_{d_{1}}(\mathbf{r})$ will intersect the domain border. Thus, $f_{1}\left(\mathbf{r}, d_{1}\right)$ is given by a piecewise function with its first piece (i.e., when $d_{1} \ll 1$ ) given by (11). The second piece (i.e., when $d_{1} \gg 1$ ) is given by differentiating (10) and multiplying by -1 . Such pdfs can be derived for general geometries. Fig. 2 depicts this for a circular domain of radius $R=5$ and a square domain of length $L=10$ for the receiver at different positions from the center. The derivations of the pdfs for these domains are straightforward. For more general polygons and to extend the results presented herein, the interested reader is referred to [11], [12].

Fig. 3 and 4 show the coverage probability for the receiver at different locations for a circular and square domain respectively. Results from Monte Carlo simulations are included to corroborate the analysis. We note a higher coverage probability near the border which then sharply drops as the receiver approaches the edge of the domain. This increase in coverage is due to the decreasing interference field near the domain borders. This in turn improves the connection probability, $H_{1}$, near borders [9].

The sharp decrease of the coverage probability is due to a lower $f_{1}\left(\mathbf{r}, d_{1}\right)$ for $\mathbf{r}$ close to the border and $d_{1} \ll 1$ as seen in Fig. 3 and 4. We also note that in the system model considered, the coverage probability is much lower when the receiver is 


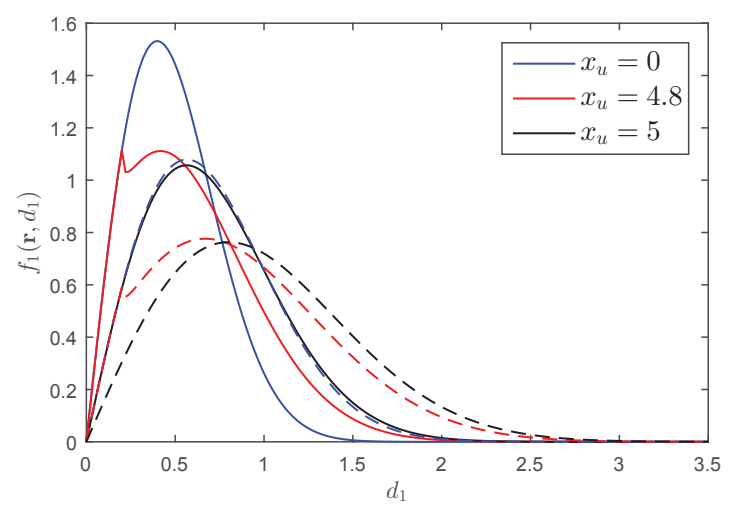

Fig. 2. Pdf of nearest neighbor in a circle (solid lines) with $R=5$ and square (broken lines) with $L=10$ domains; for the square domain, $y_{u}=5$.

located near the center of the domain. The reason for this is that the aggregate interference as observed by a receiver in this position is large. This is an intuitively satisfying result since one can imagine that a receiver located in the center of the domain sees a large number of interfering devices, whereas one located nearer to the border sees fewer interferers (but also fewer APs to which it can associate).

The results indicate an annular region close to the boundaries of the domain where higher coverage is achieved. From a system's design and optimization perspective, this leads to the conclusion that the indoor placement of additional nodes in an existing network must be within such regions. It is noteworthy that such additional deployment does not require a priori knowledge of the existing nodes locations, thus facilitating network expansion. One can further infer from the results that due to the higher interference and lower coverage at the center, additional mechanisms are necessary in the network to support a desired quality of service at any location. For instance in a dense AP deployment with a large number of users, a solution would be to assign nodes in the center of the domain a different channel (frequency) than those outside the inner regions. As such, the observed interference would decrease. The exact methods to achieve such improvement in coverage by changing the system parameters are beyond the scope of this paper. The reader should nonetheless bear in mind that the theory presented in this paper can be readily used to improve the performance of large indoor networks for instance.

\section{A. Analysis for Large Domains}

A closer inspection of (9) reveals that the sharp increase and fall of $C_{1}(\mathbf{r})$ near the border of the circular domain (c.f., Fig. 3) are due to local effects and do not depend on the domain details away from $\mathbf{r}$. This motivates us to derive an expression which can be used for large domains where domain details away from $\mathbf{r}$ can be ignored. Large here is relative to the communication length scale defined through $\left(\frac{\mathcal{P}}{q \sigma^{2}}\right)^{\frac{1}{\eta}}$. Hence we require that $d_{\max } \gg\left(\frac{\mathcal{P}}{q \sigma^{2}}\right)^{\frac{1}{\eta}}$. It is worth pointing out the large domain analysis presented herein is also useful in modeling communication systems operating at very high frequency, e.g., mmWave systems, where the communication range is limited, i.e., where local effects are dominant.

We demonstrate the approach for the positive half plane in two dimensions $\mathcal{V}=\mathbb{R}^{+} \times \mathbb{R}$. Let $\mathbf{r}=(x, 0)$. It follows that

$$
\begin{gathered}
-\frac{1}{\rho} \ln \mathcal{L}_{\mathcal{I}_{1}}\left(s_{1}\right)=2 \int_{\bar{\theta}}^{\frac{\pi}{2}} \phi\left(\frac{x}{d_{1} \cos \theta}\right) \mathrm{d} \theta-2 \phi\left(d_{1}\right) \arcsin \frac{x}{d_{1}} \\
+\pi \frac{s_{1} d_{1}^{2-\eta}}{\eta-2}{ }_{2} F_{1}\left(1,1-\frac{2}{\eta}, 2-\frac{2}{\eta},-\frac{s_{1}+\epsilon}{d_{1}^{\eta}}\right),
\end{gathered}
$$

where $\bar{\theta}=\arccos \frac{x}{d_{1}}$ when $x<d_{1}$ and 0 otherwise. It should be noted that the last term in (12) diverges for $\eta=2$ and is thus only valid for $\eta>2$. This requirement is unnecessary for finite domains $\mathcal{V}$. It is also straightforward to show that the pdf of the distance to the nearest AP is given by

$$
f_{1}\left(x, d_{1}\right)= \begin{cases}2 \rho \pi d_{1} e^{-\rho \pi d_{1}^{2}} & \text { for } d_{1}<x \\ -\frac{\mathrm{d}}{\mathrm{d} d_{1}} \hat{f}\left(x, d_{1}\right) & \text { for } d_{1} \geq x,\end{cases}
$$

where $\hat{f}_{1}\left(x, d_{1}\right)=e^{-\rho\left(\pi d_{1}^{2}+x \sqrt{\left(d_{1}-x\right)\left(d_{1}+x\right)}-d_{1}^{2} \arccos \frac{x}{d_{1}}\right)}$.

The topmost curve in Fig. 3 illustrates the coverage probability for a circular domain of radius $R=5$ and a semiinfinite domain, respectively, with $\eta=4$. Appropriate shifts and reflections are applied for the semi-infinite domain results to match the border of a circular domain since in the large domain analysis, $r=0$ corresponds to the receiver being at the border of the region as opposed to being in the center as considered in the previous sections. Note that the results agree with each other.

\section{Coverage with Cooperating AP}

In this section, we extend the previous analysis to the case where $K>1$ APs cooperatively transmit to the receiver. For the sake of analysis, we assume a joint transmission scheme where all $K$ APs communicate to the receiver using MRT. We further assume ideal conditions, such as perfect synchronization, for the joint transmission to be feasible. It should be borne in mind nonetheless that the analysis presented below is also valid for non-coherent joint transmission as justified in Appendix A of [8].

The SINR at the receiver in this case is given by

$$
\operatorname{SINR}_{K}=\frac{\mathcal{P} \sum_{k=1}^{K}\left(\left|h_{k}\right|^{2} g\left(d_{k}\right)\right)}{\sigma^{2}+\gamma \mathcal{I}_{K}},
$$

where $\mathcal{I}_{K}=\mathcal{P} \sum_{j=K+1}^{N_{\mathcal{V}}-K}\left|h_{j}\right|^{2} g\left(d_{j}\right)$. Conditioning on the location of the $K$ nearest APs, the connection probability $H_{K}$ is given by

$$
\begin{aligned}
& H_{K}\left(\mathbf{r}, d_{1}, \cdots d_{K}\right)=\mathbb{P}\left[\operatorname{SINR}_{K} \geq q \mid \mathbf{r}, d_{1}, \cdots, d_{K}\right] \\
& =\mathbb{P}\left[\sum_{k=1}^{K} g\left(d_{k}\right)\left|h_{k}\right|^{2} \geq \frac{q\left(\sigma^{2}+\gamma \mathcal{I}_{K}\right)}{\mathcal{P}} \mid \mathbf{r}, d_{1}, \cdots, d_{K}\right] .
\end{aligned}
$$

It is known that a sum of exponentially distributed random variables, each with different means follows a hypoexponential 


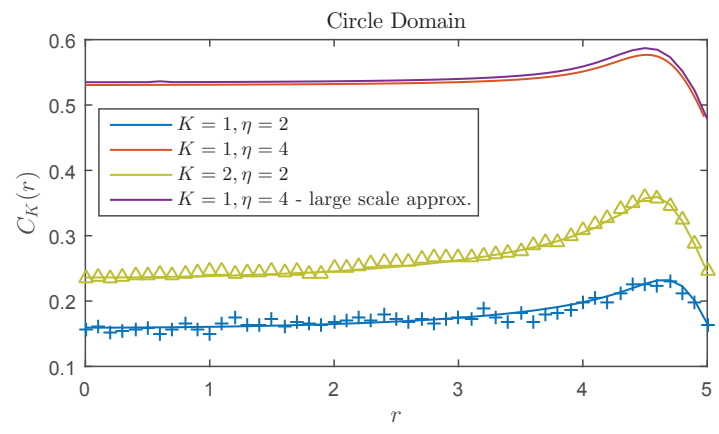

Fig. 3. Coverage probability for circular domain with $R=5$; markers represent Monte Carlo simulations.

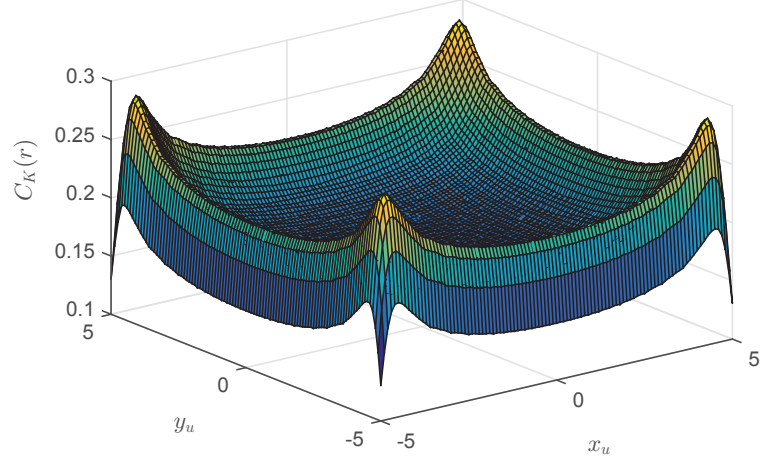

Fig. 4. Coverage probability for square domain with $L=10$.

distribution (also known as the generalized Erlang distribution). Therefore, treating $\sum_{k=1}^{K} g\left(d_{k}\right)\left|h_{k}\right|^{2}$ as a sum of $K$ independent exponentially distributed random variables with means $1 / g\left(d_{k}\right), k=1, \cdots, K$, we have that

$H_{K}\left(\mathbf{r}, d_{1}, \ldots, d_{K}\right)=\sum_{i=1}^{K}\left(\prod_{j \neq i} \frac{g\left(d_{i}\right)}{g\left(d_{i}\right)-g\left(d_{j}\right)}\right) \mathbb{E}_{\mathcal{I}_{K}}\left[e^{-\frac{x}{g\left(d_{i}\right)}}\right]$

with

$$
\mathbb{E}_{\mathcal{I}_{K}}\left[e^{-\frac{x}{g\left(d_{i}\right)}}\right]=e^{-\frac{q \sigma^{2}}{\mathcal{P} g\left(d_{i}\right)}} \exp \left(-\rho \int_{\hat{\mathcal{V}}_{K}} \frac{s_{i} g\left(d_{m}\right)}{1+s_{i} g\left(d_{m}\right)} \mathrm{dt} \mathbf{t}_{m}\right),
$$

where $\hat{\mathcal{V}}_{K}=\mathcal{V} \backslash B_{d_{K}}(\mathbf{r})$.

It is worth mentioning that the useful and interfering signals in the SINR expression above can be approximated to Gamma random variables as done in [7], [8]. However, such approximations do not provide simpler expressions for the bounded systems investigated in this paper. As such, we do not include the results using the Gamma approximation herein.

The coverage from the $K$ nearest APs under a joint transmission scheme is a functional of $H_{K}$ obtained by averaging over the distances $d_{1}, \ldots, d_{K}$. For the sake of juxtaposition, we consider the case of $K=2$. This requires the pdf $f_{2}\left(\mathbf{r}, d_{2}\right)$ of the distance to the second nearest AP given $r \in \mathcal{V}$. It follows that

$$
C_{2}(\mathbf{r})=\int_{0}^{\infty} \int_{0}^{d_{2}} H_{2}\left(\mathbf{r}, d_{1}, d_{2}\right) f_{1}\left(\mathbf{r}, d_{1}\right) f_{2}\left(\mathbf{r}, d_{2}\right) \mathrm{d} d_{1} \mathrm{~d} d_{2}
$$

where $f_{2}\left(\mathbf{r}, d_{2}\right)$ is obtained by differentiating

$$
\begin{aligned}
\mathbb{P}\left[d_{i} \geq d_{2},\right. & \forall i \geq 2]=\mathbb{P}\left[\text { at most } 1 \text { AP closer than } d_{2}\right] \\
& =\left(1+\rho\left|B_{d_{2}}(\mathbf{r}) \cap \mathcal{V}\right|\right) e^{-\rho\left|B_{d_{2}}(\mathbf{r}) \cap \mathcal{V}\right|}
\end{aligned}
$$

with respect to $d_{2}$ and multiplying the result by -1 .

Generalizing to $K$ collaborating APs, we have

$$
\begin{aligned}
C_{K}(\mathbf{r})= & \int_{0}^{\infty} \ldots \int_{0}^{d_{3}} \int_{0}^{d_{2}} H_{k}\left(\mathbf{r}, d_{1}, \cdots, d_{K}\right) \\
& \times f_{1}\left(\mathbf{r}, d_{1}\right) \ldots f_{K}\left(\mathbf{r}, d_{K}\right) \mathrm{d} d_{1} \mathrm{~d} d_{2} \ldots \mathrm{d} d_{K}
\end{aligned}
$$

where $f_{k}\left(\mathbf{r}, d_{k}\right)$ is obtained by differentiating

$$
\begin{gathered}
\mathbb{P}\left[d_{i} \geq d_{k}, \forall i \geq k\right]=\mathbb{P}\left[\text { at most } k-1 \text { AP closer than } d_{k}\right] \\
=\sum_{i=0}^{k-1} \frac{\left(\left|B_{d_{k}}(\mathbf{r}) \cap \mathcal{V}\right|\right)^{i}}{i !} e^{-\rho\left|B_{d_{k}}(\mathbf{r}) \cap \mathcal{V}\right|}
\end{gathered}
$$

with respect to $d_{k}$ and multiplying by -1 .

Plots of $C_{K}(r)$ for $K=1,2$ are shown in Fig. 3. This figure clearly indicates the benefits of cooperative transmission in the network which results in a higher coverage probability. It is interesting to note that the general shape of the coverage probability curve is similar for different $K$. This observation indicates that while cooperation improves the coverage probability, the artefacts at the borders as well as at the center of the domain still exist when MRT or joint-transmission is used. Such observations can be used by the system designer to adapt the system parameters as we mentioned in the previous section. Based on the available resources, computational complexity and latency requirements, the APs can opt to cooperate using MRT. For a desired coverage probability, our analysis can be used to deduce the minimum number of cooperative transmitters that are required at a given position in the domain. Such results can be evaluated offline and accessed when required. Although we have limited our discussion to MRT and jointtransmission in this paper, it should be borne in mind that other schemes such as coordinated zero-forcing beamforming in bounded domains can also be analyzed using a similar approach. We leave the analysis of such techniques for future work.

\section{CONCLUSION}

In this paper, we have analyzed the connectivity and coverage of an interference limited system in general confined geometries, where a receiver associates to the AP nearest to it. Using tools from stochastic geometry, we have derived expressions which demonstrate how the location of the receiver relative to the domain border affects network performance. From such expressions, the most appropriate transmission schemes and parameters can be deduced. For instance, given the observation of higher interference at the center of the domain, adequate mechanisms can be set in place to ensure that nodes in the central regions are assigned orthogonal 
resources. The analysis is further extended to the case where multiple APs associate with a given receiver where each AP sends the same signal using non-coherent joint transmission or use MRT. The framework we presented in this paper for collaborating APs can be readily extended to cater for more advanced signal processing techniques, which will be addressed in later submissions. With regards to the cooperative schemes analyzed, we conclude that while the coverage can be improved with multiple cooperating transmitters, the geometry of the domain still heavily impacts the performance of the system. From the results, the number of cooperating transmitters required at a given position to attain a target performance can be readily inferred.

\section{ACKNOWLEDGMENT}

This work was supported in part by EPSRC grant number EP/N002350/1 (Spatially Embedded Networks).

\section{REFERENCES}

[1] E. Hossain and M. Hasan, "5G cellular: key enabling technologies and research challenges," Instrumentation Measurement Magazine, IEEE, vol. 18, pp. 11-21, June 2015.

[2] N. Bhushan, J. Li, D. Malladi, R. Gilmore, D. Brenner, A. Damnjanovic, R. Sukhavasi, C. Patel, and S. Geirhofer, "Network densification: the dominant theme for wireless evolution into 5G," Comm. Mag., IEEE, vol. 52, pp. 82-89, Feb. 2014

[3] G. Mao and B. Anderson, "Towards a better understanding of largescale network models," IEEE/ACM Transactions on Networking (TON), vol. 20, no. 2, pp. 408-421, 2012.

[4] K. Hosseini, W. Yu, and R. S. Adve, "A stochastic analysis of network MIMO systems," IEEE Transactions on Signal Processing, vol. 64, pp. 4113-4126, Aug. 2016.

[5] S. Banani, A. Eckford, and R. Adve, "Analyzing the impact of access point density on the performance of finite-area networks," Communications, IEEE Transactions on, vol. 63, pp. 5143-5161, Dec 2015.

[6] G. Nigam, P. Minero, and M. Haenggi, "Coordinated multipoint joint transmission in heterogeneous networks," IEEE Transactions on Communications, vol. 62, pp. 4134-4146, Nov. 2014.

[7] J. Liu, M. Sheng, T. Q. S. Quek, and J. Li, "D2D enhanced coordinated multipoint in cloud radio access networks," IEEE Transactions on Wireless Communications, vol. 15, pp. 4248-4262, June 2016.

[8] R. Tanbourgi, S. Singh, J. G. Andrews, and F. K. Jondral, "A tractable model for noncoherent joint-transmission base station cooperation," IEEE Transactions on Wireless Communications, vol. 13, pp. 49594973, Sept. 2014.

[9] O. Georgiou, S. Wang, M. Bocus, C. Dettmann, and J. Coon, "Location, location, location: Border effects in interference limited ad hoc networks," in Modeling and Optimization in Mobile, Ad Hoc, and Wireless Networks (WiOpt), 2015 13th International Symposium on, pp. 568-575, May 2015.

[10] J. G. Andrews, F. Baccelli, and R. K. Ganti, "A tractable approach to coverage and rate in cellular networks," Communications, IEEE Transactions on, vol. 59, no. 11, pp. 3122-3134, 2011.

[11] Z. Khalid and S. Durrani, "Distance distributions in regular polygons," IEEE Transactions on Vehicular Technology, vol. 62, pp. 2363-2368, Jun 2013.

[12] R. Pure and S. Durrani, "Computing exact closed-form distance distributions in arbitrarily shaped polygons with arbitrary reference point." http://www.mathematica-journal.com/data/uploads/2015/06/Durrani.pdf. 